\title{
Experimental measurements of the spectral absorption coefficient of pure fused silica optical fibers
}

Travis J. Moore

Brigham Young University - Provo, travisjmoore@gmail.com

Matthew R. Jones

Brigham Young University - Provo, mrjones@byu.edu

Follow this and additional works at: https://scholarsarchive.byu.edu/facpub

Part of the Engineering Commons

\section{Original Publication Citation}

Travis J. Moore and Matthew R. Jones, "Experimental measurements of the spectral absorption coefficient of pure fused silica optical fibers," Appl. Opt. 54, 1374-1378 (2015)

\section{BYU ScholarsArchive Citation}

Moore, Travis J. and Jones, Matthew R., "Experimental measurements of the spectral absorption coefficient of pure fused silica optical fibers" (2015). Faculty Publications. 3156.

https://scholarsarchive.byu.edu/facpub/3156 


\title{
Experimental measurements of the spectral absorption coefficient of pure fused silica optical fibers
}

\author{
Travis J. Moore* and Matthew R. Jones \\ Department of Mechanical Engineering, Brigham Young University, 435 Crabtree Building, Provo, Utah 84602, USA \\ ${ }^{*}$ Corresponding author: travisjmoore@gmail.com
}

Received 1 October 2014; revised 16 December 2014; accepted 14 January 2015; posted 14 January 2015 (Doc. ID 223842); published 0 MONTH 0000

\begin{abstract}
Knowledge of the spectral absorption coefficient of fused silica optical fibers is important in modeling heat transfer in the processes and applications in which these fibers are used. An experimental method used to measure the spectral absorption coefficient of optical fibers is presented. Radiative energy from a blackbody radiator set at different temperatures is directed through the optical fibers and into an FTIR spectrometer. Spectral instrument response functions are calculated for different fiber lengths. The ratios of the slopes of the instrument response functions for the different lengths of fibers are used to solve for the spectral absorption coefficient of the fibers. The spectral absorption coefficient of low $\mathrm{OH}$ pure fused silica optical fibers is measured between the wavelengths 1.5 and $2.5 \mu \mathrm{m}$. (c) 2015 Optical Society of America

OCIS codes: $\quad$ (060.2400) Fiber properties; (060.2310) Fiber optics; (300.6300) Spectroscopy, Fourier transforms.

http://dx.doi.org/10.1364/AO.99.099999
\end{abstract}

\section{Introduction}

Knowledge of the spectral absorption coefficient of fused silica optical fibers is important in modeling heat transfer in the processes and applications in which these fibers are used. These include optical fiber thermometers [1-6], the splicing of optical fibers using lasers [7], the fabrication of fiber couplers and tapers [8], and the manufacture of optical fibers by drawing [9-13]. Myers [14] introduced a two-band model of the spectral absorption coefficient of fused silica that has been widely used to model heat transfer in optical fiber drawing processes [9-12]. This model neglects absorption at wavelengths less than $3 \mu \mathrm{m}$. However, Wei et al. [13] showed that the absorption in the short wavelength region is not negligible.

Various methods have been used to measure the absorption coefficient of fused silica. Izawa et al.

1559-128X/15/070001-01\$15.00/0

(C) 2015 Optical Society of America measured the spectral absorption coefficient of fused silica between wavelengths of 2.5 and $25 \mu \mathrm{m}$ using a scanning grating spectrometer $[15,16]$. Yoshida et al. estimated the absorption coefficient of fused silica at a wavelength of $1.06 \mu \mathrm{m}$ by fitting thermal lensing measurements using a Shack-Hartmann wave-front detector to a model based on the temperature dependencies of the refractive index and the thermal expansion coefficient [17]. However, no measurements have been reported over the spectral band between 1.5 and $2.3 \mu \mathrm{m}$.

Here, the spectral absorption coefficient of low $\mathrm{OH}$ pure fused silica optical fibers is measured between the wavelengths of 1.5 and $2.5 \mu \mathrm{m}$ by directing radiative energy from a blackbody radiator at different temperatures through the optical fibers and into an FTIR spectrometer. Spectral instrument response functions are created for different fiber lengths. The ratios of the slopes of the spectral instrument response function for the different lengths of fibers are used to solve for the spectral absorption coefficient of the fibers. 


\section{Experimental Setup}

The experimental setup used to measure the spectral absorption coefficient of optical fibers is shown in Fig. 1.

Radiative energy from a blackbody calibration source [18] was directed into an optical fiber through a reflective collimator [19]. The collimator consists of a $90^{\circ}$ off-axis parabolic mirror with a protected silver coating that collects collimated radiant energy and directs it into the end of the optical fiber. The average reflectance of the mirror is greater than $96 \%$ between the wavelengths of 0.45 and $20 \mu \mathrm{m}$. The fiber connects to the collimator with an SMA connector. The other end of the optical fiber was connected by an SMA connector to another reflective collimator, which collimates the radiation from the fiber and directs it into the external access port of an FTIR spectrometer [20]. The FTIR spectrometer collects the radiant energy and outputs a spectral signal. The optical fibers used in this research are multimode step-index low $\mathrm{OH}$ fibers with a $400 \mu \mathrm{m}$ diameter pure fused silica core, a $440 \mu \mathrm{m}$ diameter fluorine doped fused silica cladding, a $470 \mu \mathrm{m}$ diameter polyimide coating, and a $700 \mu \mathrm{m}$ diameter tefzel jacket [21]. The fibers have a numerical aperture of 0.22 .

\section{Instrument Response Function}

The FTIR spectrometer was equipped with a deuterated triglycine sulfate (DTGS) pyroelectric detector. Infrared radiation incident on the detector increases the detector temperature, which changes the dielectric constant of the deuterated triglycine sulfate. The change in capacitance with temperature is measured as a voltage across the detector element [22]. The spectral response of a DTGS detector has been shown to be linear [22-25]. The spectral irradiation incident on the detector, $\bar{G}_{\lambda, D}$, is related to the detector signal, $M_{\lambda}$, as follows:

$$
G_{\lambda, D}=A_{\lambda} M_{\lambda}+B_{\lambda}
$$

The offset term, $B_{\lambda}$, includes radiation emitted by the collecting optical system and by the detector itself [22-25]. The spectral irradiation incident on the detector is $[26]$

$$
G_{\lambda, D}=\int_{2 \pi} I_{\lambda, D} \cos \theta_{D} \mathrm{~d} \Omega
$$

where $I_{\lambda . D}$ is the spectral radiative intensity incident on the detector, and $\theta_{D}$ is the angle between the

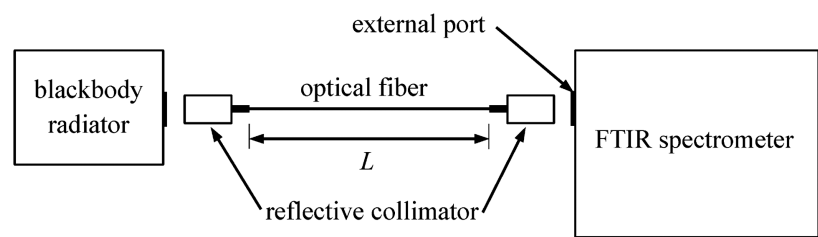

F1:1 Fig. 1. Schematic of the experimental setup used to measure the F1:2 spectral absorption coefficient of optical fibers. incident ray and the normal to the detector surface. Assuming that the collimated radiation from the reflective collimator into the FTIR spectrometer is the only source of radiative energy and that the solid angle subtended by the collimator when viewed from the detector, $\Delta \Omega_{D \rightarrow C}$, is small, Eq. (2) reduces to

$$
G_{\lambda, D} \approx I_{\lambda, D} \Delta \Omega_{D \rightarrow C} .
$$

The spectral intensity incident on the detector is directly proportional to the spectral intensity exiting the fiber,

$$
I_{\lambda, D}=P_{\lambda} I_{\lambda, f},
$$

where $P_{\lambda}$ represents attenuation along the optical path due to reflections and transmission through components in the collimator and the FTIR. An equation representing the spectral intensity exiting the fiber, $I_{\lambda, f}$, can be obtained by solving the radiative transfer equation (RTE) in the fiber. Figure 2 shows a schematic of the coordinate system used to determine $I_{\lambda, f}$.

The fiber is assumed to be an emitting, absorbing, nonscattering medium. In order to account for the multiple reflections of a beam which occur within an optical fiber, it is easier to consider the intensity at a given point $z$ in the $\hat{s}$ direction from a point source in the fiber as that coming from an image of this point projected onto the boundary a distance $z_{s}$ from the reception point, as illustrated in Fig. 2 [27]. Therefore, the optical fiber can be treated as a one-dimensional medium of length $L$.

An integrating factor is used to solve the RTE for the spectral intensity in the positive direction, $I_{\lambda}^{+}$, for which $0<\theta<\pi / 2$, and for that in the negative direction, $I_{\lambda}^{-}$, for which $\pi / 2<\theta<\pi$. The intensity at $z=0$ is the blackbody intensity coming from the blackbody radiator. The intensity at $z=L$ is that which is reflected from the interface between the fiber and the air. The details of this solution can be found in [28]. The spectral radiative intensity exiting the fiber is equal to the portion of the intensity at $z=L$ which is transmitted through the end of the fiber. It is shown in the following equation:

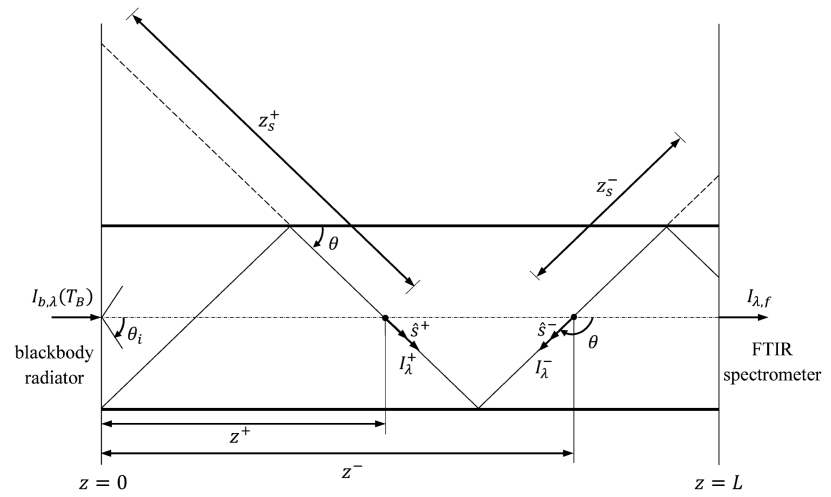

Fig. 2. Coordinate system used to determine the spectral intensity exiting the fiber. 


$$
\begin{aligned}
I_{\lambda, f}= & \tau_{\lambda, L} \int_{\mu_{i}}^{1}\left(I_{b, \lambda}\left(T_{B}\right) e^{-\kappa_{\lambda} L / \mu}\right. \\
& \left.+\frac{\kappa_{\lambda}}{\mu} \int_{0}^{L} I_{b, \lambda}\left(T\left(z^{\prime}\right)\right) e^{-\kappa_{\lambda}\left(L-z^{\prime}\right) / \mu} \mathrm{d} z^{\prime}\right) \mathrm{d} \mu .
\end{aligned}
$$

In Eq. (5), $\tau_{\lambda, L}$ is the spectral transmittance at the right boundary of the optical fiber. The first term in the parentheses of Eq. (5) represents the spectral blackbody intensity coming from the blackbody radiator at the blackbody radiator temperature, $T_{B}$. This radiative energy decreases along the length of the optical fiber due to absorption, where $\kappa_{\lambda}$ is the spectral absorption coefficient of the fiber, and $L$ is the length of the fiber. The second term in parentheses represents the contribution to the total incident intensity on the detector due to emission from the fiber. This is dependent upon the temperature profile along the fiber, $T(z)$. Both of these terms are integrated over the direction cosine, $\mu=\cos \theta$, between $\cos \theta_{i}$ and $\cos 0=1$ where $\theta_{i}$ is the critical angle that forms the acceptance cone inside of which total internal reflection occurs in the fiber. The angle $\theta$ is measured from the fiber axis. In the experimental setup used here, the entire fiber was at room temperature, so the integral on the right hand side of Eq. (ㅁ) is easily calculated.

$$
\begin{aligned}
I_{\lambda, D}= & P_{\lambda} \tau_{\lambda, L} \int_{\mu_{i}}^{1}\left(I_{b, \lambda}\left(T_{B}\right) e^{-\kappa_{\lambda} L / \mu}\right. \\
& \left.+I_{b, \lambda}\left(T_{\infty}\right)\left(1-e^{-\kappa_{\lambda} L / \mu}\right)\right) \mathrm{d} \mu .
\end{aligned}
$$

Substituting Eq. (ㅁ) into Eq. (3), substituting the result into Eq. (ㅅ) and rearranging gives

$$
I_{b, \lambda}\left(T_{B}\right)=C_{\lambda} M_{\lambda}+D_{\lambda}
$$

where

$$
C_{\lambda}=\frac{A_{\lambda}}{P_{\lambda} \tau_{\lambda, L} \Delta \Omega_{D \rightarrow C} \int_{\mu_{i}}^{1} e^{-\kappa_{\lambda} L / \mu} \mathrm{d} \mu},
$$

and

$$
\begin{aligned}
D_{\lambda}= & \frac{B_{\lambda}}{P_{\lambda} \tau_{\lambda, L} \Delta \Omega_{D \rightarrow C} \int_{\mu_{i}}^{1} e^{-\kappa_{\lambda} L / \mu} \mathrm{d} \mu} \\
& -\frac{I_{b, \lambda}\left(T_{\infty}\right)}{P_{\lambda} \tau_{\lambda, L} \Delta \Omega_{D \rightarrow C} \int_{\mu_{i}}^{1} e^{-\kappa_{\lambda} L / \mu} \mathrm{d} \mu} \int_{\mu_{i}}^{1}\left(1-e^{-\kappa_{\lambda} L / \mu}\right) \mathrm{d} \mu .
\end{aligned}
$$

In Eq. (7), $C_{\lambda}$ and $D_{\lambda}$ are the terms of the instrument response function for the experimental setup shown in Fig. 1. These are dependent on the length of the fiber. For a given fiber length, the instrument response function can be determined by collecting spectral measurements, $M_{\lambda}$, with the FTIR spectrometer at various blackbody radiator temperatures. The spectral blackbody intensity can be calculated using the Planck function [26] and linear curves fit to these intensities as a function of $M_{\lambda}$ [28]. For each wavelength, the slope of the linear fit is given by $C_{\lambda}$, and the offset is given by $D_{\lambda}$.

\section{Experiments}

The spectral instrument response function was determined using the process described above for four different lengths of optical fibers: $0.5,1,1.5$, and $2 \mathrm{~m}$. For each fiber length, spectral measurements were collected using the FTIR spectrometer at six blackbody radiator temperatures between $500^{\circ} \mathrm{C}$ and $1200^{\circ} \mathrm{C}$. Each spectrum collected by the FTIR spectrometer represents an average of 32 scans at a resolution of $32 \mathrm{~cm}^{-1}$. In order to account for variability in the experiments, at each blackbody temperature each of the fibers was secured between the two reflective collimators, and the measurements were repeated five times. The average of these five signals at each temperature was used to create the instrument response function for each fiber length.

The ratio of the slope terms, $C_{\lambda}$, of the instrument response function given in Eq. (8) for two different fiber lengths $L_{1}$ and $L_{2}$ is

$$
\frac{C_{\lambda, 1}}{C_{\lambda, 2}}=\frac{\frac{A_{\lambda}}{P_{\lambda} \tau_{\lambda, L} \Delta \Omega_{D \rightarrow C} \int_{\mu_{i}}^{1} e^{-\kappa_{\lambda} L_{1} / \mu} \mathrm{d} \mu}}{A_{\lambda}} .
$$

The $P_{\lambda}$ and $\Delta \Omega_{D \rightarrow C}$ terms are functions of the optical setup of the experiment, and the $A_{\lambda}$ term is a function of the detector. Each of these terms remains constant throughout the experiment. Therefore, if each of the fibers is assumed to have the same spectral absorption coefficient and acceptance cone, Eq. (10) can be simplified to

$$
\frac{C_{\lambda, 1}}{C_{\lambda, 2}}=\frac{\int_{\mu_{i}}^{1} e^{-\kappa_{\lambda} L_{2} / \mu} \mathrm{d} \mu}{\int_{\mu_{i}}^{1} e^{-\kappa_{\lambda} L_{1} / \mu} \mathrm{d} \mu} .
$$

The only unknown in Eq. (11) is the spectral absorption coefficient of the fiber, $\kappa_{\lambda}$. The spectral absorption coefficient can be determined from Eq. (11) using optimization methods. The least squares norm of the difference between the left side and the right side of Eq. (11) is minimized by adjusting the values of $\kappa_{\lambda}$ at the wavelengths at which measurements are made. The integrals on the right side of Eq. (11) are evaluated numerically.

The solver application in Microsoft Excel was used to find the maximum likelihood estimate of the spectral absorption coefficient in Eq. (11) using the ratios of the slopes of the instrument response function of every combination of the four fibers.

\section{Results and Discussion}

The average spectral absorption coefficient of the optical fibers calculated from all of the experiments is shown in Fig. 3. The error bars represent one standard deviation from the average. The spectral 


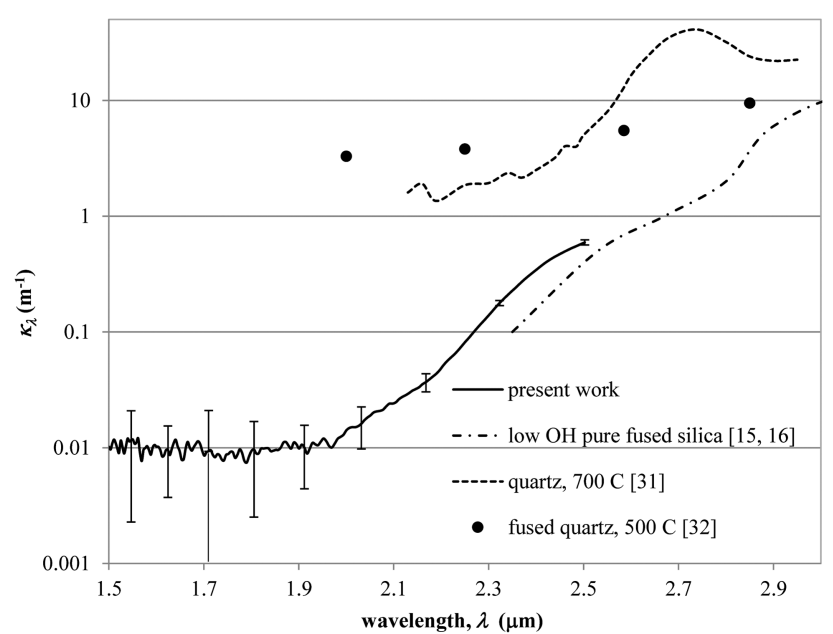

F3:1 Fig. 3. Measurements of the spectral absorption coefficient of low F3:2 OH fused silica optical fibers, low $\mathrm{OH}$ fused silica $[15,16]$, quartz F3:3 [31], and fused quartz [32]. measurements were made between $1.5 \mu \mathrm{m}$ (the lower limit of the detector) and $2.5 \mu \mathrm{m}$ (the upper limit of the transmission range of the fibers). The refractive index of silica is relatively constant over this spectral band [29]. Therefore, in Eq. (11), it was assumed that the critical angle does not vary with wavelength. Figure 3 also shows spectral absorption coefficient measurements of low $\mathrm{OH}$ fused silica made by Izawa and co-workers [15,16], which compare favorably with those made in this work. The measurements made in this work are also consistent with the measured value of $0.0018 \mathrm{~m}^{-1}$ at $1.06 \mu \mathrm{m}$ made by Yoshida et al. [17] and the reported value of $0.001 \mathrm{~m}^{-1}$ at $1 \mu \mathrm{m}$ [30].

For reference, measurements of the spectral absorption coefficients of quartz at $700^{\circ} \mathrm{C}$ [31], and fused quartz at $500^{\circ} \mathrm{C}$ [32] are also shown in Fig. 3. All data shown in Fig. $\overline{3}$ were found from published data using data extraction software. The absorption coefficient of the low $\mathrm{OH}$ fused silica fibers measured here follows the same trends as that of the quartz and fused quartz but is significantly lower. This may be a result of the water content in the glass or the fact that the measurements in [31] and [32] were made at elevated temperatures. Moisture present in the manufacturing process results in hydroxyl groups chemically bonded to the silica network ( $\mathrm{SiOH})$, which increase the transmission loss in the fibers [33]. Additionally, as shown by research performed on fused quartz, the spectral absorption coefficient of glasses increases with increasing temperature [32,34].

\section{Conclusion}

An experimental method used to measure the spectral absorption coefficient of optical fibers was presented in which radiative energy from a blackbody radiator is directed through the optical fibers and into an FTIR spectrometer. Spectral instrument response functions are calculated for different fiber lengths, and the ratios of the slopes of these functions for the different lengths of fibers are used to estimate the spectral absorption coefficient of the fibers. The spectral absorption coefficient of low $\mathrm{OH}$ pure fused silica optical fibers was measured between 1.5 and $2.5 \mu \mathrm{m}$. The spectral absorption coefficient of low $\mathrm{OH}$ pure fused silica has not previously been reported between 1.5 and $2.3 \mu \mathrm{m}$. The measurement technique is relatively simple and may be readily used to measure spectral absorption coefficients in other fibers of interest. The measurements were consistent with previous results reported between 2.3 and $2.5 \mu \mathrm{m}$.

\section{References}

1. R. R. Dils, "High-temperature optical fiber thermometer," J. Appl. Phys. 54, 1198-1201 (1983).

2 . W. S. Cheung, "The development of an optical fibre thermometer for gas turbine engines," Sens. Actuators 19, 105-117 (1989).

3. M. R. Jones and D. G. Barker, "Use of blackbody optical fiber thermometers in high temperature environments," J. Thermophys. Heat Transfer 16, 306-312 (2002).

4. D. G. Barker and M. R. Jones, "Temperature measurements using a high-temperature blackbody optical fiber thermometer," ASMEJ Heat Transfer 125, 471-477 (2003).

5. D. G. Barker and M. R. Jones, "Inversion of spectral emission measurements to reconstruct the temperature profile along a blackbody optical fiber thermometer," Inverse Probl. Eng. 11, 495-513 (2003).

6. D. J. Frankman, B. W. Webb, and M. R. Jones, "Investigation of lightpipe volumetric radiation effects in RTP thermometry," ASMEJ Heat Transfer 128, 132-141 (2006).

7. H. Fujita, Y. Suzaki, and A. Tachibaan, Method of splicing optical fibers by $\mathrm{CO}_{2}$ Laser, U.S. Patent 4263495 (April 1981).

8. T. E. Dimmick, G. Kakarantzas, T. A. Birks, and P. St. J. Russell, "Carbon dioxide laser fabrication of fused-fiber couplers and tapers," Appl. Opt. 38, 6845-6848 (1999).

9. J. Liu, S. J. Zhang, and Y. S. Chen, "Advanced simulation of optical fiber drawing process," Num. Heat Transfer Part A Applications 40, 473-795 (2001).

10. M. Taroni, C. J. W. Breward, L. J. Cummings, and I. M. Griffiths, "Asymptotic solutions of glass temperature profiles during steady optical fibre drawing," J. Eng. Math. 80, 1-20 (2013).

11. C. Chunming and Y. Jaluria, "Numerical simulation of transport in optical fiber drawing with core-cladding structure," ASMEJ Heat Transfer 129, 559-567 (2007).

12. Z. Wei, K. Lee, Z. Zhou, and S. Hong, "Modeling of advanced melting zone for manufacturing of optical fibers," J. Manuf. Sci. Eng. Trans. ASME 126, 377-387 (2004).

13. Z. Wei, K. Lee, S. W. Tchikanda, Z. Zhou, and S. Hong, "Effects of radiative transfer modeling on transient temperature distribution in semitransparent glass rod," ASMEJ Heat Transfer 125, 635-643 (2003).

14. M. R. Myers, "A model for unsteady analysis of preform drawing," AIChE J. 35, 592-602 (1989).

15. T. Izawa, N. Shibata, and A. Takeda, "Optical attenuation in pure and doped fused silica in the IR wavelength region," Appl. Phys. Lett. 31, 33-34 (1977).

16. T. Izawa and S. Sudo, Optical Fibers: Materials and Fabrication (KTK Scientific, 1987).

17. S. Yoshida, D. H. Reitze, D. B. Tanner, and J. D. Mansell, "Method for measuring small optical absorption coefficients with use of a Shack-Hartmann wave-front detector," Appl. Opt. 42, 4835-4840 (2003).

18. Landcal R1200P, www.landinst.com.

19. RC12SMA-P01, www.thorlab.us.

20. Nicolet 8700 FT-IR Spectrometer, www.thermo.com.

21. SIR400/440PIT, www.fibertech-optica.com.

22. E. Lindermeir, P. Haschberger, C. Tank, and H. Dietl, "Calibration of a Fourier transform spectrometer using three blackbody sources," Appl. Opt. 31, 4527-4533 (1992). 
23. P. C. Dufour, N. L. Rowell, and A. G. Steele, "Fourier-transform radiation thermometry: measurements and uncertainties," Appl. Opt. 37, 5923-59314 (1998).

24. L. González-Fernández, R. B. Pérez-Sáez, L. del Campo, and M. J. Tello, "Analysis of calibration methods for direct emissivity measurements," Appl. Opt. 49, 2728-2735 (2010).

25. T. J. Moore and M. R. Jones, "An experimental method for making spectral emittance and surface temperature measurements of opaque surfaces," J. Quant. Spectry. Radiative Transfer 112, 1191-1196 (2011).

26. M. F. Modest, Radiative Heat Transfer, 2nd ed., (Academic, 2003).

27. V. S. Yuferev, "Radiation-conduction heat transfer in a thin semitransparent cylinder in the light-guide approximation," J. Appl. Mech. Tech. Phys. 20, 415-418 (1980).

28. T. J. Moore, "Application of variation of parameters to solve nonlinear multimode heat transfer problems," $\mathrm{PhD}$ dissertation, Brigham Young University, Provo, UT (2014).
29. R. Kitamura, L. Pilon, and M. Jonasz, "Optical constants of silica glass from extreme ultraviolet to far infrared at near room temperature," Appl. Opt. 46, 8118-8133 (2007).

30. http://www.crystran.co.uk/optical-materials/silica-glass-sio2.

31. E. Loenen and L. van der Tempel, Determination of Absorption Coefficients of Glasses at High Temperatures, by Measuring the Thermal Emission (Philips Electronics N. V., 1996).

32. E. C. Beder, C. D. Bass, and W. F. Shackleford, "Transmissivity and absorption of fused quartz between $0.22 \mathrm{~m} 3.5 \mathrm{~m}$ from room temperature to 1500 degrees C," Appl. Opt. 10, 22632268 (1971).

33. O. Humbach, H. Fabian, U. Grzesik, U. Haken, and W. Heitmann, "Analysis of $\mathrm{OH}$ absorption bands in synthetic silica," J. Non-Cryst. Solids 203, 19-26 (1996).

34. L. V. Prikhodk and Kh. S. Bagdasarov, "Temperature dependence of IR absorption in fused quartz at high temperatures," Optika i spektroskopiâ 34, 1210-1211 (1973). 


\section{Queries}

1. AU: Should this aperture value have a unit of measurement?

2. A check of online databases revealed a possible error in this reference. The year has been changed from '1979' to '1980'. Please confirm this is correct. 